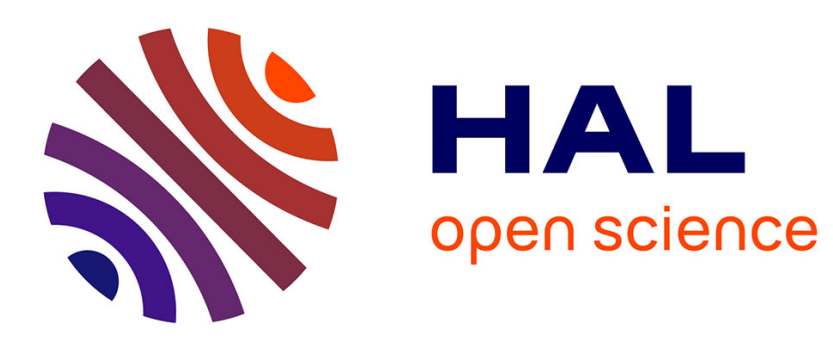

\title{
Analytical evaluation of multicast packet delivery and user clustering schemes in high-speed cellular networks
}

\author{
Neila El Heni, Xavier Lagrange
}

\section{To cite this version:}

Neila El Heni, Xavier Lagrange. Analytical evaluation of multicast packet delivery and user clustering schemes in high-speed cellular networks. Annals of Telecommunications - annales des télécommunications, 2009, 10.1007/s12243-009-0085-2 . hal-02295706

\section{HAL Id: hal-02295706 \\ https://hal.science/hal-02295706}

Submitted on 25 Nov 2019

HAL is a multi-disciplinary open access archive for the deposit and dissemination of scientific research documents, whether they are published or not. The documents may come from teaching and research institutions in France or abroad, or from public or private research centers.
L'archive ouverte pluridisciplinaire HAL, est destinée au dépôt et à la diffusion de documents scientifiques de niveau recherche, publiés ou non, émanant des établissements d'enseignement et de recherche français ou étrangers, des laboratoires publics ou privés. 


\title{
Analytical evaluation of multicast packet delivery and user-clustering schemes in high-speed cellular networks
}

\author{
Neila EI Heni • Xavier Lagrange
}

\begin{abstract}
Transmission on data-oriented radio interfaces of cellular networks has been primarily designed for unicast applications. Nevertheless, unicast may not optimize the resource usage when the same content has to be transmitted to several users in the same cell. In this context, multicast seems to be an efficient means to convey data. In this paper, we develop an analytical model that allows the computation of the mean bitrate for both multicast and multiple-unicast transmission schemes. Furthermore, we propose a multicast transmission scheme called the equal-bitrate (EB) algorithm that allocates bandwidth to mobiles according to their instantaneous channel quality. We compare it to adaptations of the well-known max-signal-to-noise ratio and round robin to multicast. We propose to group users into clusters. The clustering method combines multicast and unicast transmission schemes according to the user's average channel conditions. We use the analytical model to evaluate the proposed solutions. We compare the resulting performance against pure multicast and multiple-unicast approaches. We show that the EB algorithm offers a good trade-off between throughput and fairness. Also, we show that mixed clustering achieves good performance compared to conventional clustering methods.
\end{abstract}

Keywords Clustering • Fairness - HSDPA • Multicast transmission $\cdot$ Rayleigh fading

N. El Heni $(\varangle) \cdot X$. Lagrange Institut TELECOM, TELECOM Bretagne,

RSM Department, Université Européenne de Bretagne, Rennes BP 35510, France

e-mail: neila.elheni@telecom-bretagne.eu

\section{Introduction}

Third-generation (3G) data networks have been introduced for the support of multimedia services over wireless links. They have evolved to CDMA2000 1xEvolution Data Optimized [1] and High-Speed Downlink Packet Access (HSDPA) [2, 3], commonly known as $3.5 \mathrm{G}$ systems. The so-called 3.5 systems are enabling the delivery of very demanding applications in terms of network resource. These include video streaming such as Mobile TV [4], the VCast service [5] from Verizon, multiparty games or group-chat application, etc.

Although 3.5G networks offer high capacity, the expected demand will certainly overcome the available resources. At present, multimedia services are delivered over separate connections on the radio interface for each recipient. Packets are duplicated if there are multiple users who want to receive the same data. In this paper, such an approach is called multiple-unicast. Because spectrum is a limited and expensive resource, the radio interface can support only a handful of highbitrate users simultaneously, and the wireless link may easily become a bottleneck. In this context, multicast seems a promising alternative to conventional multipleunicast. The main benefit of multicast is bandwidth saving, as it prevents clogging up the air interface with redundant transmissions. Also, the overhead on the sender side is reduced since it does not have to maintain addresses of each destination. As commonly accepted, we define a multicast group as a set of terminals listening to the same service.

Multimedia Broadcast/Multicast Service [6, 7] is currently specified in the 3GPP recommendation to address multicast and broadcast services in UMTS/ 
WCDMA networks. Equivalently, BroadCast and MultiCast Services [8] are defined by 3GPP2 standard to support multimedia content multicast in CDMA2000 networks. However, the focus is on the access and core network rather then on the radio interface. It is implicitly assumed that transmissions are duplicated by the base station (BS) if several user equipments (UEs) in a cell belong to the same multicast group. Multicast over $3 \mathrm{G} / 3.5 \mathrm{G}$ radio interfaces is only used with low bitrate channels, which does not scale well when the number of multimedia services increases.

A common point between these networks is that they use a shared channel to serve UEs with data packets on a time-multiplexing basis. In this framework, a radio resource consists in a transmission time interval (TTI), and the scheduler's task is to share time between users of the system.

\subsection{Multiple-unicast vs multicast issues}

During a service session, users may experience different channel conditions from one TTI to another. At each TTI, the packet scheduler uses the reported signalto-noise ratio (SNR) to determine the instantaneous bitrate capacity of each user. Then, depending on its scheduling strategy, it chooses the user to serve with the suitable bitrate. This scheme is illustrated in Fig. 1. Given that the TTI is constant, determining the instantaneous bitrate capacity amounts to determining the packet size, also called the transport block size (TBS), like in HSDPA.

In multiple-unicast, user-specific adaptation of the radio parameters (e.g., to track fast fading) can be applied. As transmissions to users are considered separately, all classical scheduling mechanisms may be used, for instance, Max C/I [9], to maximize the system throughput. However, fairness has to be guaranteed between users who receive the same data. The main drawback with multiple-unicast is that a TTI is restricted to a single user. Alternatively, a TTI may be allocated to all the users listening to the same service. We call this scheme pure multicast. It is also possible to divide the multicast group into different clusters and to serve them at different times. As such, a cluster includes one or several users who listen to the same service transmission in the same TTI. If $N$ represents the number of users in the multicast group, multiple-unicast is then equivalent to $N$ single-user clusters, whereas pure multicast amounts to a single $N$-user cluster. A mixed clustering includes, for example, $N^{\prime}$ single-user clusters and a single $\left(N-N^{\prime}\right)$-user cluster. Figure 2 gives the example of two clusters.

Multicast schedulers not only have to select the cluster to serve at each TTI, but they also have the additional difficulty of determining the transmission bitrate that is common to the selected users despite their various channel conditions. Indeed, bitrate selection is a challenging task in multicast scheduling. If the transmission bitrate exceeds the bitrate capacity of a UE, this mobile will not be able to decode the transmitted packet, which is consequently lost. This is particularly true for mobiles at the cell edge. While the usage of retransmission algorithms [10] is possible to counter losses, subsequent bandwidth consumption and signaling may be costly. In all cases, the useful multicast bitrate is constrained by the UE having the worst channel, as this determines the service coverage. Hence, the gain of multicast compared to

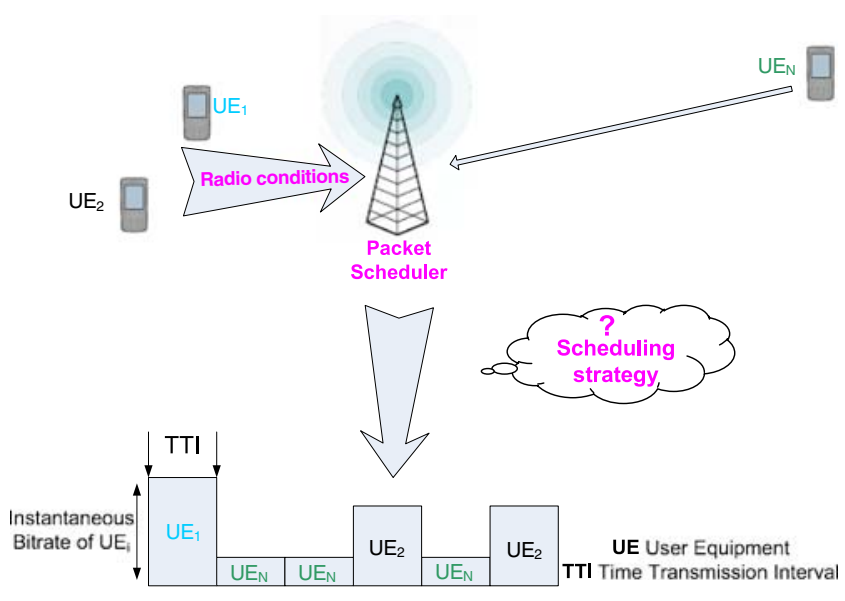

Fig. 1 Conventional unicast scheduling

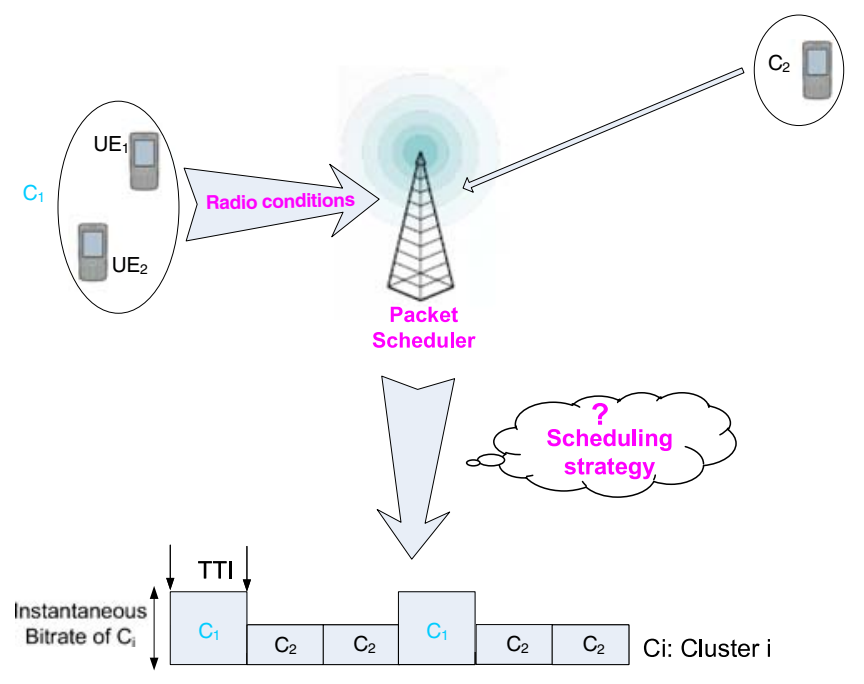

Fig. 2 Multicast transmission with an example of clustering 
multiple-unicast is not so obvious and needs to be studied. Indeed, it is difficult to predict whether it is better to duplicate data with a relatively high bitrate or to send it only once but with the lowest bitrate. Very few studies address the multicast schedulers' design. The study [11] proposes multicast proportional fairness and intergroup proportional fairness schedulers that are adaptations of the PF scheduler. However, packet loss with these algorithms may be frequent, wasting bandwidth because of retransmissions.

\subsection{Paper scope and outline}

In this study, we propose a new algorithm for multicast transmission over high-speed cellular systems and define a user clustering strategy that combines pure multicast and multiple-unicast schemes. While existing work on multicast generally addresses streaming services, our study is agnostic of the service type, which could be file transfer, video streaming, etc. We consider the transmission of one service. Scheduling multiple services amounts to managing priority between these services according to their quality-of-service (QoS) requirements. These issues have already been extensively developed in literature, e.g., [12-14].

This paper is organized as follows: In Section 2, the system model and assumptions are given. Section 3 explains the proposed equal-bitrate (EB) algorithm and presents the model for throughput calculations of max-SNR and round robin (RR). Section 4 places our model in the framework of a theoretical generic system. Particular case studies are developed in Section 5. In Section 6, we introduce state-of-the-art clustering schemes before defining a new clustering strategy. Section 7 analyzes the performance of the proposed solutions. Conclusions are drawn in Section 8.

\section{Model description}

\subsection{General assumptions}

We consider a system with one serving cell and $N$ users in the same multicast group. Dealing with multicast in different cells is equivalent to studying multicast in fixed networks, as data have to be transmitted over wired links to different BSs. This has been developed in [15]. Here, we consider downlink transmission like in a HSDPA system with only one code. Large-scale mobility aspects are not considered; signaling for clustering dynamics is, then, not developed here but may be considered as a future work.
Users are randomly distributed in the cell. To apply our multicast transmission algorithm, clusters should already be defined according to the proposed clustering strategy. Let $G$ be the number of clusters and $S_{j}$ be the size of cluster $j$. Let $\gamma_{i, j}$ be the instantaneous SNR of user device $i$, which is a member of cluster $j$, and $\overline{\gamma_{i, j}}$, its average SNR. Note that indices $i$ or $j$ may be sometimes omitted for simplicity. Due to channel variations, $\gamma_{i, j}$ are identically and independently distributed variables that change randomly from one TTI to another but are assumed to be constant during a TTI. Without loss of generality, we assume that the user having the highest average SNR is indexed by 1 and belongs to cluster 1 . Let $k_{i, j}\left(i=1 . . S_{j}, j=1 . . G\right)$ be the SNR ratios such that $k_{i, j}=\frac{\overline{\gamma_{1,1}}}{\overline{\gamma_{i, j}}}$. By definition, $k_{1,1}=1$ and $k_{i, j} \geq 1$ for $i$ and $j$ higher than 1 . Assuming the BS is serving user $i$ of cluster $j$, we define $\beta_{i, j}$ as the largest TBS supported by that user. Let $g$ be the function that relates $\beta_{i, j}$ to the reported $\gamma_{i, j}$ of the served user; hence, $\beta_{i, j}=g\left(\gamma_{i, j}\right)$. It is easy to see that $g$ is a strictly increasing function. Its associated inverse function is denoted as $h$, i.e., $\gamma_{i, j}=h\left(\beta_{i, j}\right)$.

The main notations used in this paper are listed in Table 1 . We also define $P_{X}(x)$ as the cumulative distribution function (CDF) of a random variable $X$. Hence, $P_{\gamma_{i, j}}(x)=\operatorname{Pr}\left(\gamma_{i, j} \leq x\right)$. Similarly, $p_{X}(x)$ denotes the probability distribution function (PDF) of $X$.

Table 1 List of the main notations

\begin{tabular}{ll}
\hline Notation & Meaning \\
\hline$g$ & $\begin{array}{l}\text { Function that associates a TBS } \\
\text { to the reported SNR }\end{array}$ \\
$G$ & Number of clusters \\
$i, j$ & Indices of a UE and a cluster, respectively \\
$k_{i, j}$ & SNR ratios such that $k_{i, j}=\frac{\overline{\gamma_{1,1}}}{\overline{\gamma_{i, j}}}$ \\
$F_{j}$ & Fairness factor of cluster $j$ \\
$N$ & Number of users in the cell (all belonging \\
& to the same multicast group) \\
$Q_{\text {mcast }}$ & Gain of the new clustering method \\
& compared to pure multicast \\
$Q_{\text {ucast }}$ & Gain of the new clustering method \\
$R_{j}$ & compared to multiple-unicast \\
$S_{j}$ & Mean bitrate of cluster $j$ \\
$\beta_{i, j}$ & Size of cluster $j$ \\
$\beta_{j}$ & Largest TBS supported by UE $i$, \\
$\gamma_{i, j}$ & which is member of cluster $j$ \\
& TBS of cluster $j$ \\
$\gamma_{i, j}$ & Instantaneous SNR of user $i$, \\
& which is member of cluster $j$ \\
$\gamma_{j}$ & Average SNR of user $i$, \\
\hline & which is member of cluster $j$ \\
& SNR within cluster $j$
\end{tabular}




\subsection{Propagation model}

The average SNR may be computed by using a conventional propagation model. Let $P_{i}$ be the transmit power to user $i$. The received power denoted as $P_{r}$ is, then,

$P_{r}=P_{i} h_{i} \chi_{i}$,

where $h_{i}$ is the path gain including shadowing and distance loss for user $i$ and $\chi_{i}$ is the fast fading between user $i$ and the BS. Variable $\chi_{i}$ is a random variable that represents Rayleigh fast fading. It therefore has an exponential distribution. The path gain for user $i$ at distance $r_{i}$ from the $\mathrm{BS}$ is described by

$h_{i}=10^{\frac{A_{0}}{10}} r_{i}^{\beta}$

where $\beta$ is the pathloss exponent and $A_{0}$ is the distanceloss at $1 \mathrm{~m}$ (with a BS antenna height of $30 \mathrm{~m}$, a UE antenna height of $1.5 \mathrm{~m}$, and a carrier frequency of $1,950 \mathrm{MHz})$.

The signal-to-interference ratio received by user $i$ is [16]

$\gamma_{i}=\frac{P_{i} h_{i} \chi_{i}}{\alpha\left(P_{\max }-P_{i}\right) h_{i} \chi_{i}+I_{\mathrm{ext}}}$

where $P_{\max }$ is the total transmit power of the cell, $\alpha$ is the orthogonality factor, and $I_{\text {ext }}$ represents the intercell interference. Note that $I_{\text {ext }}$ is assimilated to a constant similarly to the model given by the "enhanced UMTS radio access network extension for ns-2" (Eurane) simulator [17]. By using further modifications, we can obtain the average SNR of user $i$

$\overline{\gamma_{i}}=\int_{0}^{\gamma_{\text {sup }}} \exp \left(\frac{-I_{\text {ext }} 10^{\frac{A_{0}}{10}} r_{i}^{\beta} x}{P_{i}-\alpha\left(P_{\max }-P_{i}\right) x}\right) d x$,

where $\gamma_{\text {sup }}$ is defined by

$\gamma_{\text {sup }}=\frac{P_{i}}{\alpha\left(P_{\max }-P_{i}\right)}$.

\section{Multicast scheduling}

Multicast scheduling is performed in two steps. First, the scheduler determines the convenient transmission bitrate for each cluster. The intracluster bitrate allocation strategy is conservative. We then have

$\gamma_{j}=\min _{i=1 . . S_{j}} \gamma_{i, j}(t)$.

The CDF of $\gamma_{j}$ is equal to

$$
P_{\gamma_{j}}(x)=1-\prod_{i=1}^{S_{j}}\left(1-P_{\gamma_{i, j}}(x)\right) .
$$

Once the bitrate of each cluster is determined, the scheduler chooses the cluster to serve. In order to maximize the global throughput, a natural solution is to use max-SNR scheduling that privileges the cluster having the highest bitrate capacity. However, the scheduler must guarantee fairness between clusters. $\mathrm{RR}$ scheduler is fair with respect to system resources (i.e., time slots), but in general, the bandwidth is not shared equally among the UEs because they have different bitrate capacities. The weighted-fair queuing RR (WFQ-RR) is another variant of RR; it uses weights to achieve the same throughput for all clusters; however, WFQ-RR is agnostic of the channel conditions. In this context, we introduce the EB scheduler that realizes the same average bitrate for all the clusters while using link adaptation to address channel variations. In the next sections, we introduce our EB algorithm, then we present briefly the computation models for max-SNR and WFQ-RR.

\subsection{EB scheduler}

As all the clusters belong to the same multicast group, and in order to achieve a maximum fairness between them, EB targets the same average bitrate for all the clusters. For this purpose, we define fairness factors $\left\{F_{j}\right\}_{j=1 . . G}$ such that a cluster having a higher instantaneous bitrate capacity is served with a lower probability, i.e., the time resource is not uniformly shared between clusters. For clusters with low average channel quality, the bitrate capacity is low. Hence, in order to increase the average throughput for these clusters, their corresponding fairness factors are relatively high such that they are served more frequently. Note that, for clusters having the same average channel quality, their fairness factors are equal. At every TTI, cluster $j$ is served if the product of its instantaneous SNR and its corresponding fairness factor $F_{j}$ is the highest among all the clusters. Hence, cluster $j$ is served if and only if

$\gamma_{j} F_{j}=\max _{l=1 . . G}\left(\gamma_{l} F_{l}\right)$.

If condition 8 is fulfilled, the TBS of cluster $j$, denoted as $\beta_{j}$, is then equal to $g\left(\gamma_{j}\right)$. Otherwise, $\beta_{j}=0$. Let $\overline{\beta_{j}}\left(\gamma_{j}\right)$ be the mean TBS of cluster $j$ given that $\gamma_{j}$ is known. We have

$\overline{\beta_{j}}\left(\gamma_{j}\right)=g\left(\gamma_{j}\right) \prod_{l=1, l \neq j}^{G} P_{\gamma_{\min , l}}\left(\frac{\gamma_{j} F_{j}}{F_{l}}\right)$.

Note that if there is only one cluster, the mean TBS is equal to $g\left(\gamma_{j}\right)$. Equation 9 is still valid if the product of a null number of factors is considered equal to 1 . The 
mean bitrate of cluster $j$ denoted as $R_{j}$ is obtained by considering all the $\gamma_{j}$ values

$R_{j}=\frac{1}{D_{\mathrm{TTI}}} \int_{0}^{\infty} p_{\gamma_{j}}(x) \overline{\beta_{j}}(x) d x$

where $D_{\text {TTI }}$ is the TTI duration. Substituting Eq. 9 in Eq. 10, we obtain

$R_{j}=\frac{1}{D_{\mathrm{TTI}}} \int_{0}^{\infty} p_{\gamma_{j}}(x) g(x) \prod_{l=1, l \neq j}^{G} P_{\gamma_{\min , l}}\left(\frac{x F_{j}}{F_{l}}\right) d x$.

Equation 11 provides both a general formula for the average bitrate per cluster $j$ and a method to determine the values of $\left\{F_{j}\right\}_{j=2 . . G}$. It can be seen from Eq. 8 that only the ratio $\frac{F_{l}}{F_{j}}$ impacts the algorithm. Hence, $F_{1}$ may be arbitrarily set to 1 . As we set the average bitrate given in Eq. 11 to the same value for all the clusters, we then have a set of $G-1$ equations $R_{j}=R_{1}(j=2 . . G)$ with $G-1$ unknown values, namely, $F_{j}(j=2 . . G)$.

In an operational system, the scheduler may start by allocating the same fairness factor for all clusters and then increases this parameter gradually in clusters for which the buffer at the BS is close to saturation. A steady state is reached when all buffers have equivalent loads. Alternately, it is possible to use a lookup table at the BS to map the average SNRs to their corresponding fairness factors. Of course, that supposes a prior knowledge of the average channel conditions for each user. In the case of HSDPA, measurement reports and uplink feedbacks of the channel quality indicator may be used by the BS to estimate the average SNR.

\subsection{Max-SNR scheduler}

In order to evaluate our proposed EB scheduler, it would be interesting to compare it with reference schedulers like max-SNR or RR. Max-SNR is the most greedy scheduler with respect to throughput. At each TTI, it selects the cluster with the maximum SNR. Hence, throughput formulation can be deduced from EB scheduler for $\left\{F_{j}\right\}_{j=1 . . G}=1$.

$$
R_{j}=\frac{1}{D_{\mathrm{TTI}}} \int_{0}^{\infty} p_{\gamma_{j}}(x) g(x) \prod_{l=1, l \neq j}^{N} P_{\gamma_{l}}(x) d x .
$$

\subsection{WFQ-RR}

WFQ-RR scheduler serves users in a RR scheme without caring about their radio channel variations. It tar- gets the same throughput for all clusters. Let $\widehat{R}_{j}$ be the average bitrate capacity of cluster $j$. In other words, $\widehat{R}_{j}$ represents the throughput of cluster $j$ if it were served all the time. Hence,

$\widehat{R}_{j}=\frac{1}{D_{\mathrm{TTI}}} \mathbb{E}\left[g\left(\gamma_{j}\right)\right]$,

which is developed as follows:

$\widehat{R}_{j}=\frac{1}{D_{\text {TTI }}} \int_{0}^{\infty}\left[1-P_{\gamma_{j}}(h(x))\right] d x$.

Combining Eqs. 7 and 14, we deduce the average bitrate capacity of cluster $j$

$\widehat{R}_{j}=\frac{1}{D_{\mathrm{TTI}}} \int_{0}^{\infty} \prod_{i=1}^{S_{j}}\left[1-P_{\gamma_{i, j}}(h(x))\right] d x$.

In order to guarantee fairness among clusters with different channel conditions, the scheduler allocates to each cluster $j$ a bandwidth fraction $\rho_{j}$ such that

$\left\{\begin{array}{l}\rho_{i} \widehat{R}_{i}=\rho_{j} \widehat{R}_{j} \quad \forall i, j=1 . . G \\ \sum_{j=1}^{G} \rho_{j}=1\end{array}\right.$

After a few elementary computations, the resolution of Eq. 16 gives that

$\rho_{j}=\frac{\prod_{i \neq j}^{G} \widehat{R}_{i}}{\sum_{k=1}^{G}\left(\prod_{i \neq k}^{G} \widehat{R}_{i}\right)}$.

Then, the effective average bitrate per cluster is given by

$R_{j}=\rho_{j} \widehat{R}_{j}$

\section{Computation for a generic system}

\subsection{Generic system considerations}

We consider a generic system based on the Shannon formula [18] that gives the maximum reachable system capacity. Shannon assumes a perfect error correcting system. It allows the computation of the maximum 
bitrate depending on the SNRs. If $W$ is the available bandwidth, the maximum bitrate of user $i$ is then

$R_{\max }(\mathrm{bps})=W \log _{2}\left(1+\gamma_{i}\right)$.

The TBS is then derived as follows:

$g\left(\gamma_{i}\right)=W D_{\text {TTI }} \log _{2}\left(1+\gamma_{i}\right)$.

Next, we can deduce the associated inverse function

$h(x)=2^{\frac{x}{W D_{\mathrm{TTI}}}}-1$.

In [19], Knopp and Humblet proposed a reference radio channel model based on an exponential distribution for $\gamma$.

$p_{\gamma}(x)= \begin{cases}\frac{1}{\bar{\gamma}} \cdot \exp \left(\frac{-x}{\bar{\gamma}}\right) & \text { if } x>0 \\ 0 & \text { otherwise }\end{cases}$

where $\bar{\gamma}$ is the average received SNR. The associated $\mathrm{CDF}$ is

$P_{\gamma}(x)=1-\exp \left(\frac{-x}{\bar{\gamma}}\right)$.

Note that the SNR given in Eq. 3 does not follow an exponential distribution. However, we can verify that, when $\gamma_{\text {sup }}$ tends to infinity, the SNR has an exponential distribution. Hence, the approximation of using both Eq. 23 for the CDF of $\gamma$ and Eq. 4 for its mean value makes sense.

Assuming that the SNR of each user follows an exponential distribution, we can deduce the CDF of $\gamma_{j}$ from Eqs. 7 and 23 as follows:

$P_{\gamma_{j}}(x)=1-\exp \left(\frac{-x \sum_{i=1}^{S_{j}} k_{i, j}}{\overline{\gamma_{1,1}}}\right)$.

Its corresponding PDF is derived as follows:

$p_{\gamma_{j}}(x)=\frac{\sum_{i=1}^{S_{j}} k_{i, j}}{\overline{\gamma_{1,1}}} \exp \left(\frac{-x \sum_{i=1}^{S_{j}} k_{i, j}}{\overline{\gamma_{1,1}}}\right)$.

\subsection{EB throughput}

Substituting Eqs. 20, 24, and 25 in Eq. 11, we reformulate the EB average bitrate per cluster $j$ as follows:

$R_{j}=W \frac{\sum_{i=1}^{S_{j}} k_{i, j}}{\overline{\gamma_{1,1}}} \int_{0}^{\infty}\left[\exp \left(\frac{-x \sum_{i=1}^{S_{j}} k_{i, j}}{\overline{\gamma_{1,1}}}\right) \log _{2}(1+x)\right.$

$$
\left.\times \prod_{l=1, l \neq j}^{G}\left(1-\exp \left(\frac{-\frac{x F_{j}}{F_{l}} \sum_{i=1}^{S_{l}} k_{i, l}}{\overline{\gamma_{1,1}}}\right)\right)\right] d x .
$$

Equation 26 gives the average bitrate per cluster $j$ considering Shannon and an exponential distribution for SNR.

As multiple-unicast case corresponds to $N$ singleuser clusters, the average bitrate is derived from Eq. 26 for $G=N$ and $S_{j}=1(\forall j=1 . . G)$. We denote it as $R_{\text {ucast }}$ and we formulate it hereafter

$$
\begin{aligned}
R_{\mathrm{ucast}}= & W \int_{0}^{\infty}\left[\frac{1}{\overline{\gamma_{j}}} \cdot \exp \left(\frac{-x}{\overline{\gamma_{j}}}\right) \log _{2}(1+x)\right. \\
& \left.\times \prod_{l=1, l \neq j}^{N}\left(1-\exp \left(\frac{-\frac{x F_{j}}{F_{l}}}{\overline{\gamma_{j}}}\right)\right)\right] d x
\end{aligned}
$$

with $F_{j}$ verifying $R_{j}=R_{1}(\forall j=1 . . N)$.

In the framework of pure multicast, We denote the average bitrate as $R_{\text {mcast }}$. It is derived after a few computations including integration by parts and variable changing.

$R_{\text {mcast }}=\frac{1}{D_{\mathrm{TTI}}} \cdot \int_{0}^{\infty} \exp \frac{-\left(2^{\frac{x}{\text { W. } D_{\mathrm{TTI}}}}-1\right) \sum_{i=1}^{N} k_{i}}{\overline{\gamma_{1}}} d x$.

Note that cluster indice $j$ is omitted from $k$ and $\bar{\gamma}$ parameters as there is only one cluster.

Finally, formulations for max-SNR are deduced from EB expressions with fairness factors set to 1 .

\subsection{WFQ-RR throughput}

Considering the $(\mathrm{K} \& \mathrm{H})$ model given in Eq. 23 and the inverse of shannon formula given in Eq. 21, we reformulate Eq. 15 as follows:

$$
\widehat{R}_{j}=\frac{1}{D_{\mathrm{TTI}}} \int_{0}^{\infty} \prod_{i=1}^{S_{j}} \exp \left(\frac{-2^{\frac{x}{W D_{\mathrm{TTI}}}}+1}{\overline{\gamma_{i, j}}}\right) d x,
$$


which is equivalent to

$$
\widehat{R}_{j}=\frac{1}{D_{\mathrm{TTI}}} \int_{0}^{\infty} \exp \left(\frac{\left(-2^{\frac{x}{{ }^{\frac{D}{\mathrm{TTI}}}}}+1\right) \sum_{i=1}^{S_{j}} k_{i, j}}{\overline{\gamma_{1}}}\right) d x
$$

From Eqs. 18 and 29, the average bitrate per cluster is

$$
R_{j}=\frac{\rho_{j}}{D_{\mathrm{TTI}}} \int_{0}^{\infty} \exp \left(\frac{\left(-2^{\frac{x}{W D_{\mathrm{TTI}}}}+1\right) \sum_{i=1}^{S_{j}} k_{i, j}}{\overline{\gamma_{1}}}\right) d x
$$

\section{Case studies}

In this section, we target performance evaluation for some particular cases like equivalent users or uniform user distribution. For the sake of simplicity and due to space limitation, we restrict ourselves to the EB algorithm, first because it makes part of the contributions of this work, and second because it seems to offer a good trade-off between system throughput and user fairness. In fact, unlike WFQ-RR, this scheduler uses link adaptation to track the channel variations, and, unlike max-SNR, it realizes throughput fairness between clusters. The objective is to study, in simple examples, the behavior of our scheduler. Also, this part orients us toward a new efficient clustering scheme. The comparison with the other schedulers is addressed in a more general case in Section 6.

\subsection{Serving equivalent users}

Considering $N$ users with the same average SNR, we compare the single $N$-user and the $N$ single-user clustering schemes. We have compared these schemes in [16]; however, we take back the obtained results to justify our new clustering strategy. Let $\bar{\gamma}$ be the common average SNR value for all users. In this context, fairness factors $F_{j}$ and SNR ratios $k_{j},(j=1 . . N)$ are equal to 1 . Hence, the average unicast bitrate is deduced from Eq. 27 as follows:

$$
\begin{aligned}
R_{\text {ucast }}= & \frac{W}{\bar{\gamma}} \int_{0}^{\infty} \exp \left(\frac{-x}{\bar{\gamma}}\right) \log _{2}(1+x) \\
& \times\left(1-\exp \left(\frac{-x}{\bar{\gamma}}\right)\right)^{N-1} d x
\end{aligned}
$$

and Eq. 28 becomes

$$
R_{\mathrm{mcast}}=\frac{1}{D_{\mathrm{TTI}}} \cdot \int_{0}^{\infty}\left(\exp \frac{-\left(2^{\frac{x}{W \cdot D_{\mathrm{TTI}}}}-1\right) N}{\bar{\gamma}}\right) d x
$$

In the framework of multiple-unicast, our EB algorithm is equivalent to the opportunistic max $\mathrm{C} / \mathrm{I}$ scheduler. As users have the same average SNR, they will receive the same bandwidth fraction on average and fairness is not affected. We evaluate the multicast gain, which represents the ratio between the pure multicast bitrate and the multiple-unicast bitrate. We denote this gain as $\Gamma_{m}$. Hence,

$\Gamma_{m}=\frac{R_{\mathrm{mcast}}}{R_{\mathrm{ucast}}}$.

Simulation parameters are listed in Table 2; they are compatible with Eurane simulator [17] and coherent with our propagation model to be applied in typical urban environments. Two regions can be depicted. Figure 3 presents the multicast gain $\Gamma_{m}$ vs the average SNR for various $N$. For high average SNRs (above around $3.7 \mathrm{~dB}$ ), it is better to use multicast, for which the gain increases with $N$. In this case, bandwidth saving by transmitting packets only one time to several users has a higher impact than the bitrate limitation by the minimum SNR. It is also observed that the larger the average SNR becomes, the greater the span in system gain becomes between different values of $N$. However, when average SNRs are low (below $3.7 \mathrm{~dB}$ ), multiple-unicast becomes more attractive. In this case, it is better to take advantage from opportunistic scheduling and to select the UE that has the best SNR even if the same data are duplicated over several times to the different users. Moreover, the greater $N$ is, the smaller the multicast gain is. Performance evaluation for HSDPA is coherent with the generic case. Multicast can largely outperform multiple-unicast for acceptable average channel quality. When the mean SNR is high enough, minimum and maximum bitrate

Table 2 Simulation parameters

\begin{tabular}{lc}
\hline Frame period $\left(D_{\text {TTI }}\right)$ & $2(\mathrm{~ms})$ \\
BS Transmission power $\left(P_{\max }\right)$ & $38(\mathrm{dBm})$ \\
Power of Intra-cell interferants $\left(P_{\max }-P_{i}\right)$ & $30(\mathrm{dBm})$ \\
Inter-cell interference $\left(I_{\text {ext }}\right)$ & $-100(\mathrm{dBm})$ \\
$\beta$ & 3.52 \\
$A_{0}$ & $31.8(\mathrm{~dB})$ \\
$W$ & $5(\mathrm{MHz})$ \\
\hline
\end{tabular}




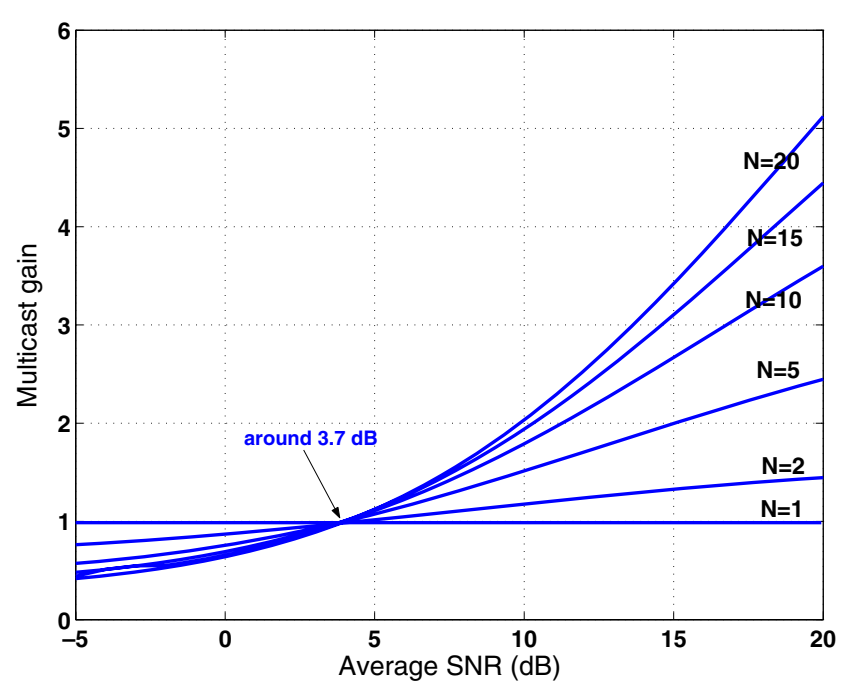

Fig. 3 Multicast gain $\Gamma_{m}$ vs $\bar{\gamma}$ for different $N$

capacities within a multicast group are equivalent, and then, as multicast serves all terminals at one TTI instead of $N$ TTIs, the multicast gain limit is

$\lim _{\bar{\gamma} \rightarrow \infty} \Gamma_{m}=N$

This gain value is also obtained for multicast in fixed networks.

\subsection{Study of a uniform user distribution}

In this section, we consider five users $\left(U E_{i=1.5}\right)$ that are uniformly distributed over a cell and sorted by their distance from the BS in an increasing order of their indices. As such, $U E_{1}, \ldots, U E_{5}$ are distant from the BS by $223,316,387,447$, and $500 \mathrm{~m}$, respectively. Their average SNRs are deduced from their respective distances from the BS as shown in Eq. 4. Other simulation parameters are listed in Table 2. The objective is to deduce in a simple example the best clustering strategy to be coupled with the EB scheduler, and also to verify coherence with results of Section 5.1. Table 3 gives the most interesting tested configurations considering different clustering schemes. Note that $F_{j}$ values are computed by numerical resolution of an equation system, as has been explained at the end of Section 3.

Configuration 1 corresponds to pure multicast where all users belong to cluster $C_{1}$. Configurations 2, 3, and 4 consider two clusters. For instance, in configuration 2, users with high $\bar{\gamma}$ values and indexed by 1,2 , and 3 are grouped in the same cluster $\left(C_{1}\right)$ and users 4 and 5 in a second cluster $\left(C_{2}\right)$. Configuration 5 considers three clusters. Finally, configuration 6 illustrates the multipleunicast case with five clusters.

We can verify in configurations 1 and 2 that grouping users with low channel quality $\left(U E_{4}\right.$ and $\left.U E_{5}\right)$ offers the worst performance (1.69 and $1.87 \mathrm{Mbps}$ ). Multipleunicast is then better for these users. On the other hand, configuration 5, which isolates users with low average SNRs while grouping together terminals with good channel quality, offers the best throughput (3.11 Mbps). This confirms the idea of using multicast for users near the BS. It is noteworthy that, instead of serving users having low average SNRs in a unicast mode, it seems interesting to cluster them with users close to the BS. In fact, as the bitrate allocation strategy to each cluster is conservative, the bitrate value is influenced by the lowest capacity within the cluster. Hence, adding a good user will have no tangible impact on the throughput. This can be verified when we look at configurations 3 and 4 in Table 3. In the former, the worst user is isolated in a separate group; in the latter, the user with the highest SNR is grouped with the worst user. The resulting performance is the same.

\section{User clustering}

Multiple-unicast and pure multicast are two extreme clustering strategies. In a previous work [16], we compared these strategies for the particular case of equivalent users, i.e., users with the same average SNR. In this study, we consider a mixed clustering strategy in a more general case where users are anywhere in the cell.
Table 3 Clustering schemes' performance for five uniformly distributed users $\left(\overline{\gamma_{1}}=24, \overline{\gamma_{2}}=9, \overline{\gamma_{3}}=5\right.$, $\overline{\gamma_{4}}=0.8$ and $\overline{\gamma_{5}}=-3 \mathrm{~dB}$ )

\begin{tabular}{|c|c|c|c|c|}
\hline \multicolumn{3}{|c|}{ Configuration } & \multicolumn{2}{|l|}{ Results } \\
\hline ID & $G$ & $\left\{U E_{1}, U E_{2}, U E_{3}, U E_{4}, U E_{5}\right\}$ & $\overline{\left\{F_{j}\right\}_{j=1 . . G}}$ & Rate (Mbps) \\
\hline 1 & 1 & $C_{1}, C_{1}, C_{1} C_{1}, C_{1}$ & $\{1\}$ & 1.69 \\
\hline 2 & 2 & $C_{1}, C_{1}, C_{1}, C_{2}, C_{2}$ & $\{1,28.4\}$ & 1.87 \\
\hline 3 & 2 & $C_{1}, C_{1}, C_{1}, C_{1}, C_{2}$ & $\{1,2.3\}$ & 2.06 \\
\hline 4 & 2 & $C_{1}, C_{2}, C_{2}, C_{2}, C_{1}$ & $\{1,1.92\}$ & 2.06 \\
\hline 5 & 3 & $C_{1}, C_{1}, C_{1}, C_{2}, C_{3}$ & $\{1,2.1,11\}$ & 3.11 \\
\hline 6 & 5 & $C_{1}, C_{2}, C_{3}, C_{4}, C_{5}$ & $\{1,59,187,680,2980\}$ & 1.96 \\
\hline
\end{tabular}




\subsection{Previous work on multicast clustering}

Existing multicast schemes introduce two clustering approaches. First, we find basic clustering that ignores QoS aspects. It includes service-oriented clustering [20], where clusters are created depending on the service availability. This means that a user subscribing to a specific multicast service is integrated into the cluster where this service is provided, independently of the user's location. If only one service is considered, this scheme is equivalent to pure multicast with one cluster. Another variant of the service-oriented clustering is given in [21]. Instead of automatically using multicast for users subscribing to the same service, authors propose to first check the number of these users and then decide the transmission mode. If this number is below a predefined threshold, multiple-unicast is adopted. Otherwise, pure multicast is used. Considering only one service, we clearly see that this clustering scheme is equivalent to either multiple-unicast or pure multicast. As basic clustering schemes do not consider the user channel quality, the deviation between the best and the worst user within a cluster may be so important that the allocation of a common bitrate inside a cluster will lack accuracy. In fact, if the chosen bitrate is high, there will be losses for users having bad radio conditions. Conversely, if the bitrate is too low, terminals with good radio quality will be penalized as their channels will be under-exploited. A second clustering approach is proposed in [20] to alleviate the aforementioned problems. It involves a QoS-oriented approach where users are grouped according to their received quality. Conceptually, UEs near the BS should belong to a cluster with better quality and faraway UEs belong to other clusters with lower quality. The division of UEs into quality groups ensures that the deviation between the best and the worst reception within a cluster is less significant. However, according to the previous section, grouping users with low SNRs decreases the system performance. For this reason, we propose a new QoSbased clustering scheme, namely, mixed clustering.

\subsection{Proposed clustering strategy}

We have seen in Section 5 that multicast is attractive only for high average SNRs. Our proposed clustering scheme is then deduced as illustrated in Fig. 4.

- An average SNR threshold is fixed so that the system can differentiate users. The average SNR is declared as low if it is below a threshold value denoted as $\bar{\gamma}_{\text {thres. }}$. Let $N_{\text {low }}$ be the number of users having a low average SNR.

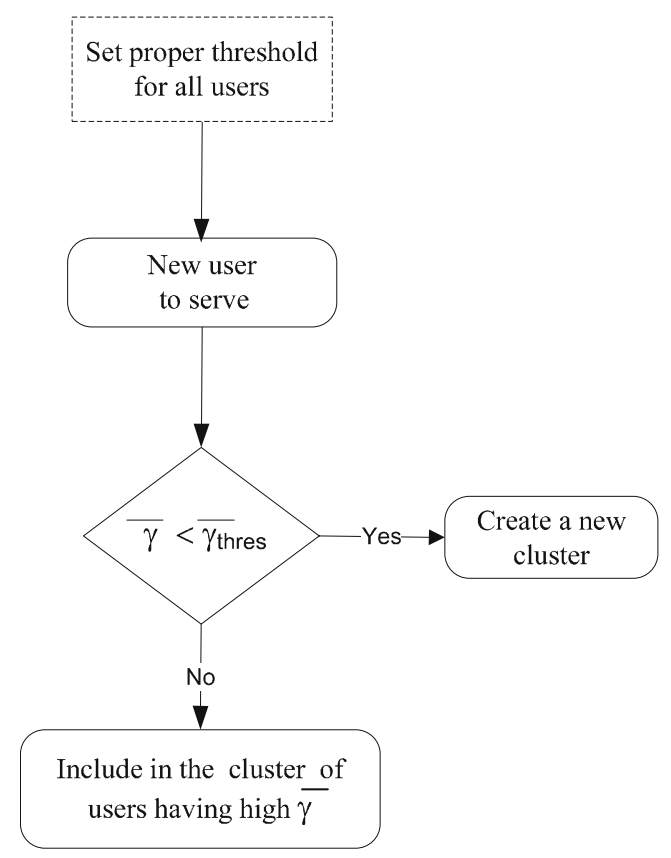

Fig. 4 Proposed clustering strategy

- Users with low average SNRs have to be separated from each other. In fact, if the cluster size increases for low SNR values, the instantaneous bitrate capacity within the cluster becomes lower, as the multicast strategy is conservative. Our solution is to serve these users according to a unicast scheme.

- Users with high average SNRs should follow a multicast scheme. They are grouped in the same cluster that contains $N-N_{\text {low }}$ users. Consequently, the resulting number of clusters $G$ is equal to $N_{\text {low }}+1$. Of course, if all users have low average channel quality, $G$ is equal to $N_{\text {low }}$.

As it combines pure multicast and multiple-unicast, our clustering scheme is called mixed clustering.

\section{Evaluation results}

In order to evaluate our mixed clustering approach, we compare it to multiple-unicast with $N$ single-user clusters and pure multicast with a single $N$-user cluster. We take $\bar{\gamma}_{\text {thres }}$ equal to $3.7 \mathrm{~dB}$, which is the threshold value depicted in Fig. 3. Section 7.3 shows that the tolerance margin for $\bar{\gamma}_{\text {thres }}$ is quite large. We consider randomly located users. Several iterations are performed until acceptable confidence intervals are achieved. Each iteration corresponds to a given user distribution. Then, the average performance is computed over all the iterations including the $95 \%$ confidence intervals. 
7.1 Performance evaluation in terms of average bitrate

We compare the throughput performance of the studied schedulers associated to different clustering schemes, namely, multiple-unicast, multicast, and mixed. For instance, considering the EB scheduler, we record in each iteration the average bitrate given by Eq. 26 for mixed clustering, by Eq. 27 for multipleunicast, and by Eq. 28 for pure multicast.

The throughput performance averaged over all the iterations for EB scheduler in comparison with maxSNR and WFQ-RR schedulers is shown in Fig. 5. We can state that, for all the schedulers, mixed clustering offers the highest performance. We can also verify that curves of pure multicast are the same for all schedulers. In fact, as pure multicast throughput deals with a single cluster, it is independent of the cluster selection scheme. Instead, it depends on the link adaptation scheme, which is conservative for all our schedulers. Dealing with the other clustering schemes, we see that max-SNR offers the highest throughputs, while the lowest performance is obtained with WFQ-RR. In fact, the latter does not take profit from link adaptation.

Figure 6 shows the ratio between the EB throughput with mixed clustering and EB throughput with conventional clustering, namely, pure multicast and multipleunicast. This gain is computed for a fixed number of terminals and different user distributions. In each distribution, the average distance between UEs and the $\mathrm{BS}$ is represented on the $\mathrm{x}$ axes. When all the users have high SNRs, our clustering solution is equivalent
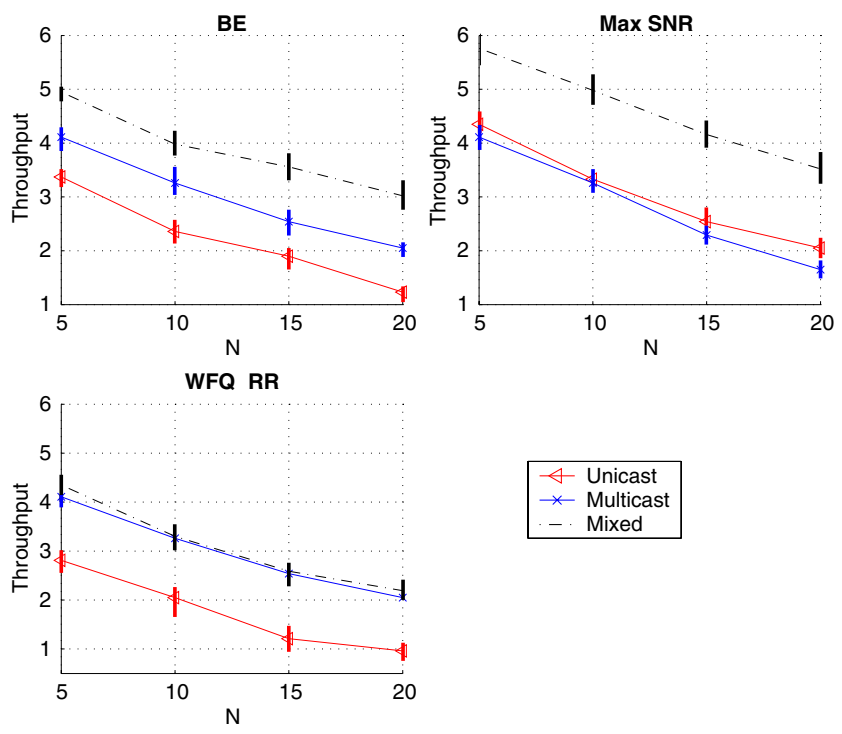

Fig. 5 Throughput (Mb/s) for the different scheduling and clustering strategies with $95 \%$ confidence intervals
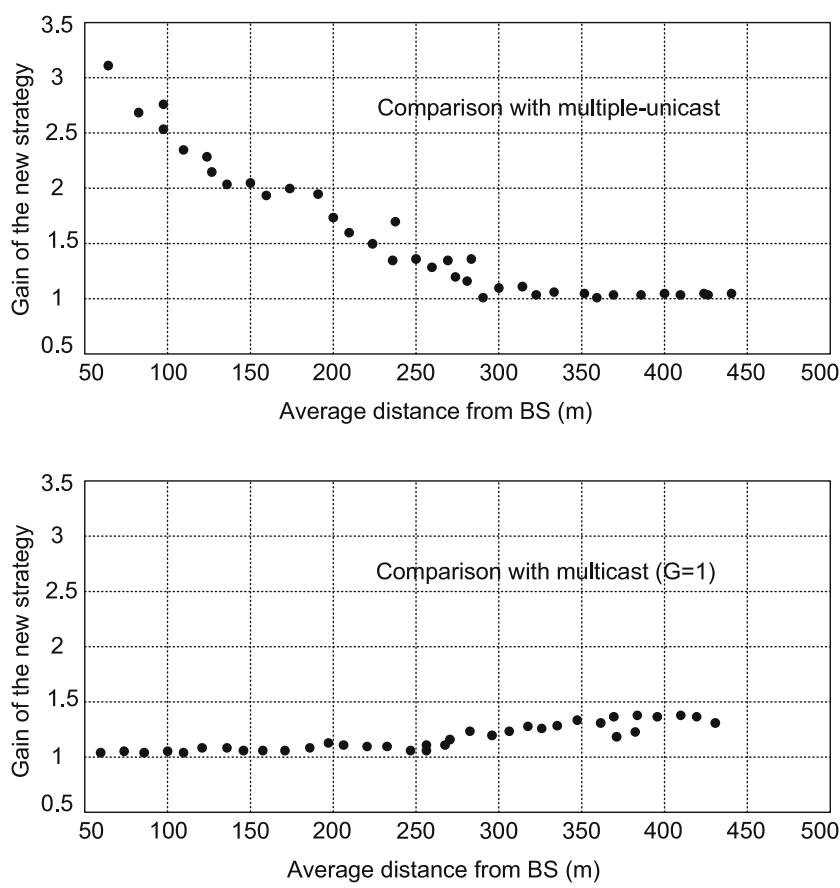

Fig. 6 Gain of the new clustering strategy compared to multicast and multiple-unicast

to pure multicast, the gain against pure multicast being equal to 1 . On the other hand, the case when all the users have low average SNRs comes down to multipleunicast as the gain against multiple-unicast is equal to 1. In the other cases where users have heterogeneous channel qualities, the EB algorithm combined with the new clustering scheme generally offers the best performance, as the gain values in Fig. 6 are always higher than 1 .

Figure 7 shows the number of clusters vs the average distance between users and the BS. For low average

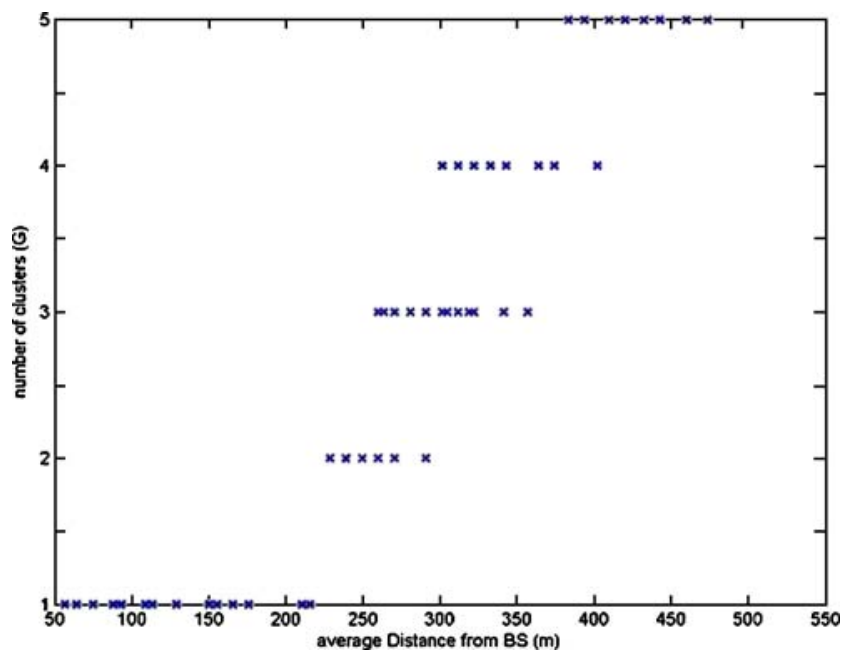

Fig. 7 Number of clusters vs the average distance from the BS 
distances from the BS, the number of clusters is equal to 1 ; hence, we verify that our strategy corresponds to pure multicast. When users get closer to the cell border, the number of clusters increases and our strategy tends to multiple-unicast.

\subsection{Fairness evaluation}

Fairness is an important issue for the scheduling design. A fair scheduler prevents the automatic resource allocation to users with a good channel quality. In order to evaluate fairness for each scheduler, we use the coefficient of variation $(\mathrm{CV})$, which is a normalized measure of the probability distribution dispersion. It is defined as the ratio of the standard deviation to the mean.

As EB and WFQ target the same throughput for all clusters, their $\mathrm{CV}$ is equal to zero for all the clustering schemes. Conversely, max-SNR scheduler results in high CV. In Fig. 8, we estimate the mean CV values for the different schedulers with multiple-unicast and mixed clustering. Of course, the $\mathrm{CV}$ is null for pure multicast. The CV is lower with mixed clustering compared to multiple-unicast, and it increases for a higher number of users. We can clearly see that, in spite of the good performance of max-SNR in terms of throughput and transmission duration, it is unfair to users with a bad channel quality. The CV reaches $28 \%$ for 15 users served in a unicast scheme.

\subsection{Impact of the average SNR threshold $\bar{\gamma}_{\text {thres }}$}

In order to see the impact of $\bar{\gamma}_{\text {thres }}$ on the new clustering scheme, we perform additional simulations for different values of this parameter. The gain is averaged over 100 iterations. Let $Q_{\text {ucast }}$ be the gain of the new clustering method compared to multiple-unicast. Hence,

$Q_{\text {ucast }}=\frac{R_{\text {mixed }}-R_{\text {ucast }}}{R_{\text {ucast }}}$,

where $R_{\text {mixed }}$ and $R_{\text {ucast }}$ are the average bitrate performances of the new clustering strategy and multiple-
Table 4 Gain of the new strategy compared to pure multicast and multiple-unicast for various $\bar{\gamma}_{\text {thres }}$ values

\begin{tabular}{lrrrrrr}
\hline $\bar{\gamma}_{\text {thres }}(\mathrm{dB})$ & \multicolumn{1}{c}{5} & 2 & 3.7 & \multicolumn{1}{l}{5} & \multicolumn{1}{l}{15} \\
\hline$Q_{\text {mcast }}$ & 5 UEs & $0 \%$ & $13 \%$ & $19 \%$ & $4 \%$ & $-18 \%$ \\
& 10 UEs & $0 \%$ & $18 \%$ & $23 \%$ & $10 \%$ & $-26 \%$ \\
& 15 UEs & $0 \%$ & $23 \%$ & $30 \%$ & $14 \%$ & $-35 \%$ \\
$Q_{\text {ucast }}$ & 5 UEs & $23 \%$ & $40 \%$ & $46 \%$ & $28 \%$ & $0 \%$ \\
& 10 UEs & $36 \%$ & $56 \%$ & $68 \%$ & $34 \%$ & $0 \%$ \\
& 15 UEs & $54 \%$ & $71 \%$ & $100 \%$ & $46 \%$ & $0 \%$ \\
\hline
\end{tabular}

unicast, respectively. Similarly, we define $Q_{\text {mcast }}$ as the gain compared to pure multicast. It is noteworthy that the performance of multiple-unicast and pure multicast is independent of $\bar{\gamma}_{\text {thres }}$. Only the bitrate performance of the new clustering scheme is influenced by $\bar{\gamma}_{\text {thres }}$ values.

Results are given in Table 4. The proposed clustering method provides the highest gain for $\bar{\gamma}_{\text {thres }}$ around $3.7 \mathrm{~dB}$, which is coherent with the value obtained in Fig. 3. For low $\bar{\gamma}_{\text {thres }}$ values $(-5 \mathrm{~dB})$, we see that the new clustering scheme is similar to pure multicast as $Q_{\text {mcast }}$ is equal to $0 \%$. In fact, in this case, all users are considered with high average channel quality and are therefore clustered together. For high $\bar{\gamma}_{\text {thres }}$ values $(15 \mathrm{~dB})$, the new clustering strategy is similar to multiple-unicast, as $Q_{\text {ucast }}$ is equal to $0 \%$. In fact, in this case, all users tend to be considered with low average SNRs and are therefore separated into different clusters. According to Fig. 5, the global performance of EB with multiple-unicast is lower than the one with pure multicast. Hence, $Q_{\text {mcast }}$ is negative when $\bar{\gamma}_{\text {thres }}$ is very high.

Except for the highest value of $\bar{\gamma}_{\text {thres }}$, the proposed clustering method always provides a gain compared to both multicast and multiple-unicast, whatever $\bar{\gamma}_{\text {thres }}$ is. Among moderate values of $\bar{\gamma}_{\text {thres }}$, the threshold of $3.7 \mathrm{~dB}$ provides the highest gain. It is noteworthy that the gain with neighboring threshold values (like 2 and $5 \mathrm{~dB})$ remains interesting. This allows an important tolerance margin for the choice of $\bar{\gamma}_{\text {thres }}$ in which the use of our clustering scheme is favorable.
Fig. $8 \mathrm{CV}$ for the different schedulers and clustering schemes
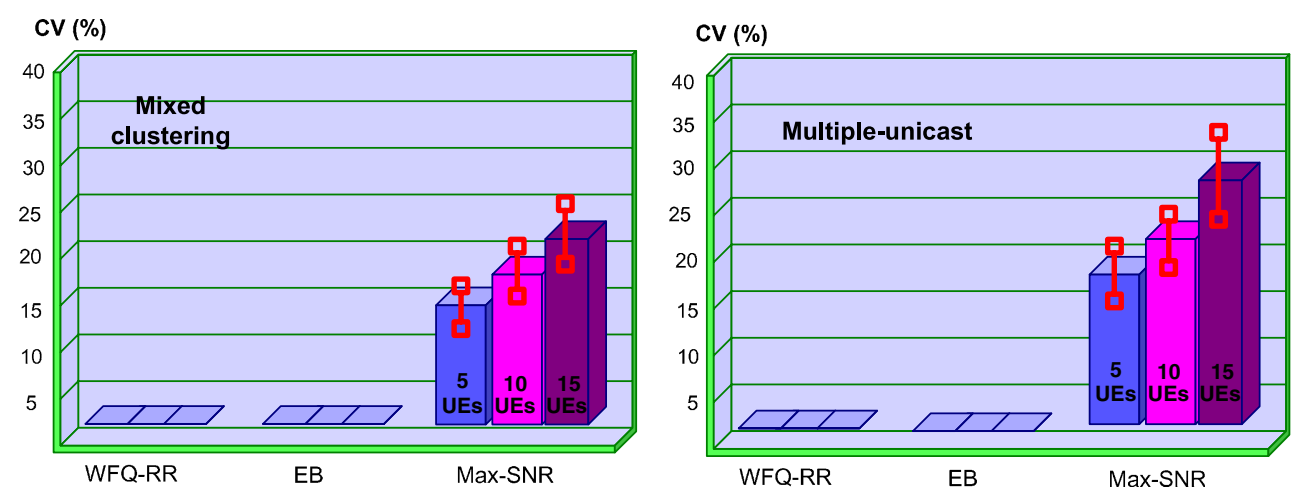


\section{Conclusion}

This study deals with multicast transmission over highspeed cellular networks. In this framework, we propose the EB algorithm, which allows fairness among clusters while offering good system throughput. We have developed an analytical model for the average bitrate calculations in order to evaluate the resulting scheduling performance and compared it with two reference schedulers, namely, max-SNR and WFQ-RR. To ensure an efficient usage of our algorithm, we have proposed to use a mixed clustering strategy. We have shown that our solution outperforms both multipleunicast and pure multicast strategies. Although the gain is influenced by the threshold value for the average SNR, a wide tolerance margin for this parameter exists in which it is always favorable to use the new clustering scheme.

The proposed solution is agnostic of the service type and may be refined by considering both radio metrics and QoS metrics. However, it is quite complicated to model these QoS metrics, and resorting to simulations seems to be necessary. This could be considered in a future work.

\section{References}

1. Bender P, Black P, Grob M, Padovani R, Sindhushyana N, Viterbi S (2000) CDMA/HDR: a bandwidth efficient high speed wireless data service for nomadic users. Commun Mag IEEE 38:70-77, Jul

2. 3GPP (2006) High speed downlink packet access (HSDPA): overall description. 3GPP TS 25.308 v7.1.0, Dec. 2006

3. Xavier LAGRANGE (2005) Principes et évolutions de l'UMTS. Hermès, Paris (collection ic2 edition)

4. MobiTV (2009) Mobile television and radio service provider. http://www.mobitv.com

5. Segan S (2005) PC Magazine Verizon's vcast: video over evdo phones, Jan. 2005. http://www.pcmag.com/article2/0, $1759,1749429,00$.asp

6. 3GPP (2007) Multimedia broadcast/multicast service (MBMS); architecture and functional description. 3GPP TS 23.246 v.8.0.1, Dec. 2007
7. 3GPP (2008) Introduction of the multimedia broadcast multicast service (MBMS) in the radio access network (RAN); stage 2. 3GPP TS 25.346 v8.1.0, March 2008

8. High rate broadcast packet data air interface specification. TIA-1006-1, Nov. 2005

9. Borst S (2005) User-level performance of channel-aware scheduling algorithms in wireless data networks. IEEE/ACM Trans Netw 13:636-647

10. Vartiainen V, Kurjenniemi J (2007) Point-to-multipoint multimedia broadcast multicast service (MBMS) performance over HSDPA. In: IEEE 18th international symposium on personal, indoor and mobile radio communications (PIMRC), 3-7 Sept. IEEE, Piscataway, pp 1-5

11. Won H, Cai $\mathrm{H}$ et al (2007) Multicast scheduling in cellular data networks. In: INFOCOM, 26th IEEE international conference on computer communications, May. IEEE, Piscataway, pp 1172-1180

12. Lundevall M, Olin B, Olsson J, Wiberg N, Wanstedt S, Eriksson J, Eng F (2004) Streaming applications over HSDPA in mixed service scenarios. In: IEEE 60th vehicular technology conference (VTC), Sept. IEEE, Piscataway, pp 841-845

13. Kazmi M, Wiberg N (2003) Scheduling algorithms for HSDSCH in a WCDMA mixed traffic scenario. In: 14th IEEE proceedings on personal, indoor and mobile radio communications (PIMRC), 7-10 Sept, vol 2. IEEE, Piscataway, pp 1485-1489

14. Braga AR, Rodrigues EB, Cavalcanti FRP (2006) Packet scheduling for VoIP over HSDPA in mixed traffic scenarios. In: IEEE 17 th international symposium on personal, indoor and mobile radio communications (PIMRC), Sept. IEEE, Piscataway, pp 1-5

15. Rummler R, Chung YW, Aghvami AH (2005) Modeling and analysis of an efficient multicast mechanism for UMTS. IEEE Trans Veh Technol 54:350-365, Jan

16. El Heni N, Lagrange X (2008) Multicast vs multiple-unicast scheduling in high-speed cellular networks. In: IEEE 62nd vehicular technology conference (VTC), Dallas, May 2008

17. Eurane (2006) Eurane website. http://www.ti-wmc.nl/eurane/

18. Shannon C (1948) A mathematical theory of communication. Bell Syst Tech J 27:379-423, 623-656, Oct

19. Knopp R, Humblet PA (1995) Information capacity and power control in single-cell multiuser communications. In: IEEE international conf. on communications, Seattle. IEEE, Piscataway, pp 331-335

20. Eusebio P, Marquet A, Martins N, Correia A (2004) Management scenarios for multicast groups in enhanced-UMTS. In: IEEE 60th vehicular technology conference (VTC), Sept, vol 4. IEEE, Piscataway, pp 3045-3049

21. Bakker H, Zeller D (2005) A method of providing a multimedia service in a digital wireless communication network. U.S. Patent EP1507423, February 